УДК 622.276 .53

\title{
ДИНАМИЧЕСКИЕ СИМУЛЯТОРЫ В ЗАДАЧАХ ДИАГНОСТИКИ ШТАНГОВЫХ ГЛУБИННО-НАСОСНЫХ УСТАНОВОК
}

\author{
Зюзев Анатолий Михайлович1, \\ a.m.zyuzev@urfu.ru
}

\author{
Самуэль Исаак Текле 1 , \\ samuel47tecle@gmail.com \\ 1 Уральский федеральный университет, \\ Россия, 620002, г. Екатеринбург, ул. Мира, 19.
}

\begin{abstract}
Актуальность. Эфффективность работы штанговых глубинно-насосных установок во многом зависит от качества системы мониторинга и диагностики неисправностей агрегата. Когда штанговый насос эксплуатируется в критических рабочих состояниях, частота отказов оборудования увеличивается, а эфффективность производства снижается. Кроме того, поскольку штанговый насос работает глубоко под землей, затраты на его ремонт и обслуживание весьма высокие, а добыча при этом прерывается на длительное время. Следовательно, улучшение системы мониторинга и диагностики работы штангового насоса является важной задачей. Инфрормация о рабочем состоянии штангового насоса отражается в динамограмме усилий в подвеске колонны штанг и в диаграмме мгновенной мощности двигателя - ваттдиаграмме. Мониторинг штангового насоса с использованием диаграммы мощности двигателя более эфффективен, чем динамометрирование, так как его можно использовать для оценки состояния как наземного, так и подземного оборудования. Кроме того, диаграмма мощности двигателя, в отличие от динамограммы, получается с помощью более простых и надежных средств измерений тока и напряжения. Таким образом, диаграмму мощности двигателя можно признать лучшим альтернативным методом для разработки оперативных систем мониторинга и диагностики для штанговых насосных установок.

Основная цель: рассмотреть проблемы построения диагностических моделей с использованием диаграммы мощности двигателя штанговых глубинно-насосных установок.

объекты: электропривод, штанговая насосная установка, нефтедобывающая скважина.

Методы: имитационное моделирование; метод извлечения признаков, который создает вектор признаков для уникального представления каждого рабочего состояния - метод опорных векторов.

Результаты. Проанализированы 72 расчётных диаграммы мощности двигателя, представляющие шесть рабочих состояний, а именно: нормальное рабочее состояние; утечка всасывающего клапана; воздействие газа; недостаточная подача жидкости; низкая и высокая посадка плунжера. Показано, что вектор признаков, построенный на основе оценки мощности в момент переключения клапанов, уникально представляет каждое рабочее состояние. Также было замечено, что метод опорных векторов правильно классифицирует образцы в нормальном режиме работы штанговых глубинно-насосных установок, утечки во впускном клапане и заполнение насоса газом. Однако некоторые образцы с недостаточной подачей жидкости были ошибочно классифицированы как заполнение насоса газом или нормальные.
\end{abstract}

\section{Ключевые слова:}

Штанговый насос, извлечение признаков, динамограмма, диагностика неисправностей

\section{Введение}

Штанговый насос является наиболее распространенной установкой механизированного подъема пластовой жидкости в нефтяной промышленности. В $[1,2]$ показано, что около $90 \%$ нефтяных скважин в США, 80 \% нефтяных скважин в мире оборудованы штанговыми глубинно-насосными установками (ШГНУ). Причины преобладания штанговых насосов связаны с простотой их использования и высокой эффективностью. Однако диагностика неисправностей и обслуживание штанговых насосов - дело дорогостоящее и сложное.

Известно более 20 различных рабочих состояний ШГНУ, которые могут быть визуально определены экспертами в данной предметной области [3]. Стоимость эксплуатации штангового насоса возрастает, если он работает в некоторых нештатных состояниях. Следовательно, создание системы эффективного мониторинга работы штангового насоса является важной задачей. Динамограмма важна для контроля рабочего состояния штангового насоса. Различные условия работы штанговых насосов можно предста- вить разными формами динамограмм [4]. Традиционно опытный технический персонал диагностирует состояние ШГНУ по виду динамограмм. Однако этот метод неэффективен, поскольку он практически исключает мониторинг в реальном времени, а на результат влияет субъективный опыт эксперта.

В ряде работ, таких как [4-7] и т. д., для повышения эффективности мониторинга ШГНУ была предпринята попытка автоматизировать систему диагностики неисправностей с использованием методов машинного обучения. Для этого предлагается выполнять извлечение характерных признаков процесса с последующей классификацией для решения задачи диагностики неисправностей. На основе средств обработки данных известные подходы можно разделить на контролируемое обучение, которое требует участия человека для обработки данных, и неконтролируемое обучение, которое позволяет обрабатывать данные без участия человека. При использовании контролируемых и неконтролируемых методов обучения предполагается, что доступные образцы соответствуют требованиям обучающей выборки. Однако 
этого трудно достичь в реальных условиях, потому что только несколько из возможных рабочих состояний возникают на ранних сроках эксплуатации скважин, а образцы из разных скважин не всегда имеют одинаковое распределение. Более того, неправильная динамометрическая карта может быть получена из-за неправильной калибровки, конфигурации или настройки датчиков положения штока, кривошипа или вала двигателя.

Диаграмму мощности двигателя предлагается использовать в качестве альтернативного источника данных для диагностики штангового насоса [8, 9]. Мониторинг штангового насоса с использованием ваттдиаграммы более эффективен, чем на основе динамограммы, так как её можно использовать для мониторинга как наземного, так и подземного оборудования. Например, состояние балансировки агрегата однозначно отражается на диаграмме мощности двигателя [10]. Кроме того, ваттдиаграмма получается с помощью более простых средств измерения тока и напряжения двигателя. Однако следует отметить, что не существует признанной связи между диаграммой мощности двигателя и диагностируемыми состояниями, хотя эффекты всех возможных состояний подземной и наземной частей ШГНУ отражаются в диаграмме мощности двигателя в соответствующие моменты времени.

Образцы диагностируемых состояний, созданные с помощью компьютерной модели, могут решить проблемы, связанные с требованиями к обучению и трудностями при маркировке диаграмм мощности двигателя. Модель ШГНУ можно разделить на модель наземной части привода, модель движения колонны штанг с плунжерным насосом и модель геологической среды [11]. Многомассовое последовательно-распределённое представление колонны насосных штанг и НКТ описывает их возвратно-поступательное движение [12]. Решение модели движения колонны насосных штанг определяет состояние штангового насоса, при этом параметры жидкости в скважине существенно влияют на результаты решения задачи.

\section{Устройство и анализ работы штанговой глубинно- насосной установки}

В состав оборудования штангового насоса входит приводной двигатель, редуктор, наземное насосное оборудование, колонна насосных штанг, насоснокомпрессорных труб (НКТ) и собственно плунжерный насос. В большинстве ШГНУ в качестве источника движущего усилия применяется электродвигатель, момент от которого через клиноременную передачу и редуктор передаётся на вал кривошипного механизма [13]. В наземной части насосной установки используется четырёхплечий шарнирный механизм для преобразования вращательного движения кривошипа в возвратно-поступательное движение насосных штанг. Собственно насос состоит из плунжера в корпусе и клапанного механизма, обеспечивающего преобразование возвратно-поступательного движения плунжера в поступательное движение жидкости.
Динамическое поведение штангового насоса сложный процесс, в котором задействовано множество взаимозависимых переменных. Клапанный механизм насоса содержит всасывающий (приточный) и нагнетательный (напорный) клапаны. Работа этих клапанов связана с возвратно-поступательным движением полированного штока, передающего движение плунжеру через колонну штанг, и может быть понята из рис. 1, на котором приведены типичные диаграммы перемещения полированного штока $\left(\mathrm{S}_{\mathrm{pht}}\right)$, скорости $\left(\mathrm{v}_{\mathrm{pht}}\right)$, нагрузки $\left(\mathrm{F}_{\mathrm{pht}}\right)$, давления насоса $\left(\mathrm{p}_{\mathrm{HAC}}\right)$ и кривой мощности двигателя (Р) на одном обороте кривошипа. В нормальном рабочем состоянии нагнетательный клапан закрывается в конце хода вверх, а всасывающий клапан закрывается в конце хода вниз. Однако открытие обоих клапанов зависит от количества свободного газа в насосе. Давление насоса, определяющее нагрузку на плунжер, зависит от состояния этих клапанов. Когда всасывающий клапан открыт, давление насоса почти равно давлению жидкости в скважине и к плунжеру с системой штанг прикладывается только выталкивающая сила. В то время как при открытом нагнетательном клапане давление насоса почти равно давлению нагнетания и на плунжер оказывается большая нагрузка.

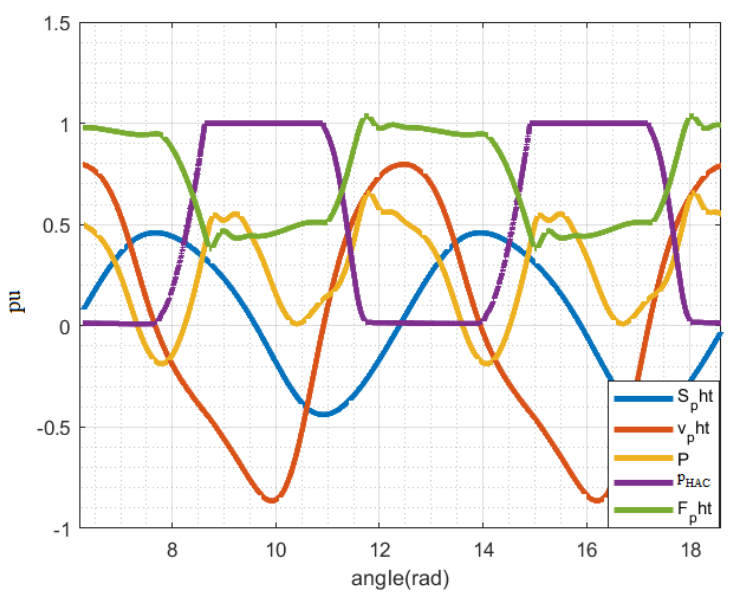

Pис. 1. Типичные диаграммы механизма

Fig. 1. Typical curves for mechanism analysis

Влияние неравномерной нагрузки на плунжер в цикле качания вызывает периодическое изменение нагрузки на редуктор. Поэтому в большинстве штанговых насосов на кривошипе закреплен противовес для выравнивания воздействия неравномерных нагрузок и уменьшения мощности приводного двигателя. Во время хода штока вниз противовес поднимается, и его потенциальная энергия увеличивается, напротив, во время движения штока вверх противовес опускается, возвращая механизму накопленную энергию. Следовательно, противовес помогает двигателю поднимать вес колонны насосных штанг с жидкостью во время хода вверх и, наоборот, во время хода вниз вес колонны насосных штанг помогает двигателю поднимать противовес. Чередование и расположение экстремумов на диаграмме мощности двигателя может 
быть использовано для анализа состояния рабочего процесса штангового насоса.

Проблемы мониторинга штанговых глубинно-насосных установок

Использование надежной системы мониторинга, диагностики неисправностей и средств автоматического управления необходимо для поддержания работы штангового насоса в нормальном рабочем состоянии, поскольку его эксплуатация в критических режимах может привести к увеличению частоты отказов оборудования или снижению его производительности. Большинство частных критических рабочих состояний ШГНУ связано с проблемами в подземной части оборудования. Усилие в колонне насосных штанг реагирует на воздействие этих проблем, отображаясь в мгновенной мощности приводного двигателя. По этой очевидной причине наземная динамограмма была первым инструментом для мониторинга подземной части штангового насоса. Известно, что информация о рабочих состояниях штангового насоса содержится в форме динамограммы. Например, замкнутая кривая вида параллелограмма характеризует нормальное рабочее состояние насоса; выпуклость в нижнем левом углу характеризует состояние, при котором плунжер ударяется в корпус насоса в нижней мертвой точке (низкая посадка плунжера); параболическая кривая в верхней части характеризует эффект утечки всасывающего клапана; наоборот, нисходящая дуга означает эффект протечки нагнетательного клапана; на кривой недостаточной подачи жидкости (неполное заполнение насоса) отсутствует правый нижний угол. Таким образом, анализ формы динамограммы без прямого доступа к подземному оборудованию позволяет определить, в каком состоянии находится штанговый глубинный насос.

Основная проблема с традиционным методом диагностики на основе динамометрической карты (динамограммы) связана с затратами рабочего времени и необходимыми знаниями и опытом в данной области. Кроме того, серьезные проблемы возникают в связи с надежностью и точностью существующих систем динамометрирования. Одним из способов получения динамограмм является использование синхронизированных измерений нагрузки и перемещения полированного штока ШГНУ. Для измерения нагрузки обычно используется проволочный тензодатчик, сконфигурированный по полной мостовой схеме, устанавливаемый в траверсу подвески полированного штока. Диагностика этим методом имеет низкую надежность при стационарной установке датчика, а при выполнении периодического контроля необходимо останавливать агрегат и фиксировать полированный шток для установки датчика. Кроме того, весьма вероятный небольшой дисбаланс мостовой схемы изменяет профиль динамометрической карты. Некорректные динамограммы также получаются из-за неправильной калибровки датчиков измерения положения полированного штока.

Формирование динамограммы на основе электрических измерений потребляемой электродвигателем мощности - еще один способ её получения. Хотя надежность электрических измерений гарантируется, точность расчётной динамограммы снижается, как правило, из-за некорректной конфигурации геометрии насосного агрегата. По сравнению с динамограммой электрические измерения содержат больше полезной информации о состоянии наземной и подземной части ШГНУ. Например, качество балансировки, которое является решающей информацией при вводе в эксплуатацию штанговых насосов, не может быть выявлено напрямую с помощью динамограммы [14]. Однако состояние баланса можно визуально наблюдать на кривой мощности двигателя, сравнивая два пика, имеющихся в одном цикле откачки. Поскольку жесткость механических характеристик двигателя довольно высокая, нагрузка на полированный шток возрастает в точках, близких к нижней и верхней мертвой точке. Важным свойством диаграммы мощности двигателя ШГНУ является её цикличность с периодом, равным времени двойного хода полированного штока. Таким образом, эффекты изменения нагрузки насоса периодически повторяются и отражаются на диаграмме мощности двигателя в соответствующие моменты времени. Это указывает на многообещающий потенциал использования диаграммы мощности двигателя для мониторинга и диагностики состояния штанговых глубинных насосов. Однако при разработке диагностической модели штангового насоса на основе оценки мгновенной мощности возникают определённые проблемы, препятствующие разработке эффективной системы мониторинга и диагностики.

Рабочий цикл подземного насоса весьма сложен. Масса и объем газа в насосе непрерывно изменяются в зависимости от таких переменных, как перемещение плунжера и уровень жидкости в скважине. Из-за этих особенностей работы штангового насоса доступные для контроля переменные не могут гарантировать точность оценки рабочего состояния. Кроме того, возможно, что одни и те же состояния насоса могут быть вызваны разными причинами [15]. Таким образом, следует провести более глубокое исследование по выбору характерных признаков, которые лучше всего описывают рабочий процесс ШГНУ.

Модель классификации для системы диагностики выводится из набора обучающих данных. В процессе обучения требуется множество помеченных образцов, чтобы гарантировать точность эффекта распознавания. Однако маркировка данных требует специальных знаний и является затратным по времени процессом. Кроме того, состояние штангового насоса непрерывно изменяется, при этом в течение срока службы скважины возникает лишь несколько рабочих состояний из множества возможных. Следовательно, трудно выполнить требования по обучению на реальной установке.

\section{Динамический симулятор для штанговых насосов}

На рис. 2 представлена структурная схема имитационной модели ШГНУ. Переменными процесса являются приток жидкости, забойное давление, давление всасывания, давление нагнетания, скорость истечения, нагрузка на плунжер, перемещение плунжера, 
скорость плунжера, перемещение полированного штока, нагрузка на полированный шток. Подробное пояснение блоков на рис. 2 представлено в следующих разделах.

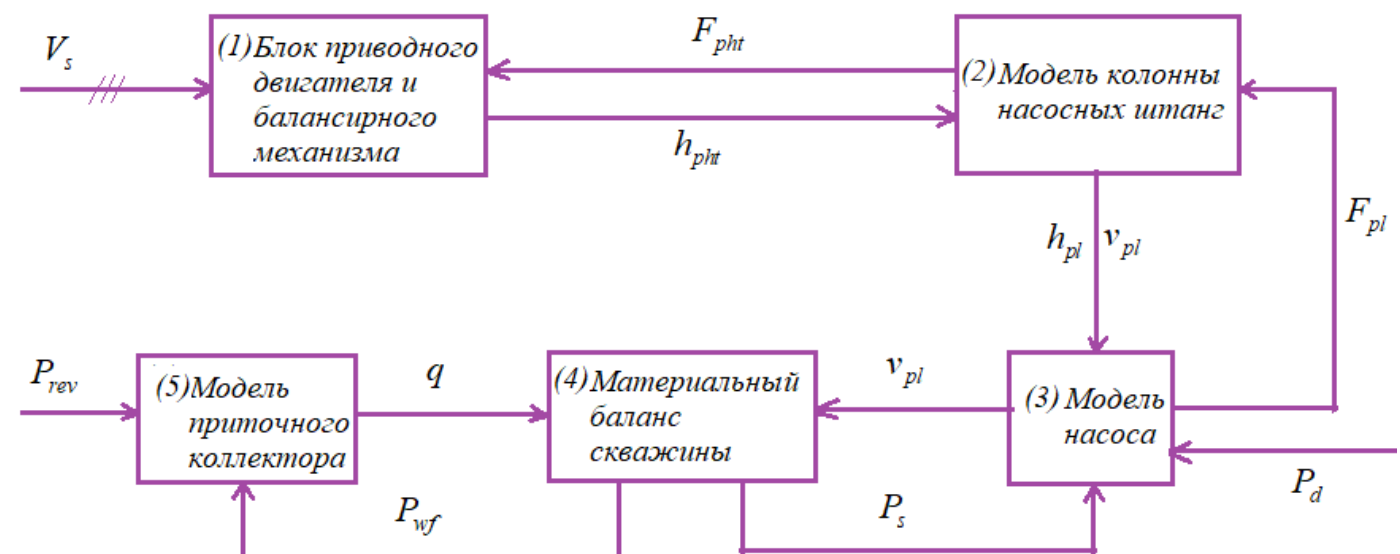

Pис. 2. Имитационная модель штангового насоса. $V_{s}-$ напряжение питания; $F_{p h t}-$ нагрузка на полированный шток; $h_{p h t}$ - перемещение полированного штока; $h_{p l}$ - перемещение плунжера; $v_{p l}$ - скорость плунжера; $F_{p l}$ нагрузка на плунжер; $P_{r e v}$ - пластовое давление; $P_{w f}-$ забойное давление; $P_{s}-$ давление всасывания; $P_{d}$ - давление нагнетания; $q$ - скорость потока жидкости

Fig. 2. Sucker rod pump simulation model. $V_{s}$ - supply voltage; $F_{p h t}$ - polished rod load; $h_{p h t}$ - polished rod displacement; $h_{p l}$ - plunger displacement; $v_{p l}$ - plunger velocity; $F_{p l}$-load on plunger; $P_{\text {rev }}$ - reservoir pressure; $P_{w f}$-bottomhole pressure; $P_{s}-$ suction pressure; $P_{d}$-discharge pressure; $q$-liquid flow rate. 1 -prime mover and pumping unit model; 2 -sucker rod string model; 3 - pump model; 4 -material balancing, 5 -reservoir model

\section{Модель колонны насосных штанг}

Колонна насосных штанг соединяет плунжер подземного насоса с наземным оборудованием. Её длина укорачивается или удлиняется в зависимости от нагрузки на плунжер насоса. Такое поведение позволяет волнам усилия распространяться по её длине. Авторы в [16] использовали одномерное уравнение затухающей волны для аппроксимации движения колонны насосных штанг. Колонну насосных штанг также можно представить как пружинно-демпферную систему, состоящую из распределённых по длине системы элементов «пружина-масса-демпфер» [17] Система уравнений (1) более применима для моделирования поведения колонны насосных штанг [12].

$$
\left\{\begin{array}{l}
m_{1} \ddot{u}_{1}=k_{o}\left(u_{o}-u_{1}\right)-k_{1}\left(u_{2}-u_{1}\right)+ \\
\quad+c_{o}\left(\dot{u}_{o}-\dot{u}_{1}\right)-c_{1}\left(\dot{u}_{2}-\dot{u}_{1}\right) ; \\
m_{2} \ddot{u}_{1}=k_{1}\left(u_{1}-u_{2}\right)-k_{2}\left(u_{3}-u_{2}\right)+ \\
\quad+c_{1}\left(\dot{u}_{1}-\dot{u}_{2}\right)-c_{2}\left(\dot{u}_{3}-\dot{u}_{2}\right) ; \\
\vdots \\
\quad m_{n-1} \ddot{u}_{n-1}=k_{n-2}\left(u_{n-2}-u_{n-1}\right)-k_{n-1}\left(u_{n}-u_{n-1}\right)+ \\
\quad+c_{n-2}\left(\dot{u}_{n-2}-\dot{u}_{n-1}\right)-c_{n-1}\left(\dot{u}_{n}-\dot{u}_{n-1}\right) ; \\
m_{n} \ddot{u}_{n}=k_{n-1}\left(u_{n-1}-u_{n}\right)+c_{n-1}\left(\dot{u}_{n-1}-\dot{u}_{n}\right)-F_{p}(t),
\end{array}\right.
$$

где $c_{i}$ - коэффициент жесткости; $k_{i}$ - коэффициент демпфирования элемента; $m_{i}-$ масса элемента, $u_{i}-$ перемещение элемента; $F_{p}$ - усилие на плунжере насоса.

\section{Блок приводного двигателя и балансирного механизма}

Современные системы балансирных приводов штанговых насосов состоят из преобразователя элек- трической энергии, электродвигателя, клиноременной передачи, шестеренчатого редуктора и четырехзвенного шарнирного механизма сдвоенного типа. В качестве приводного двигателя широко используется асинхронный двигатель. Система уравнений, описывающая динамику асинхронного двигателя в переменных состояния «ток статора $\left(i_{s}\right)$ - поток ротора $\left(\psi_{s}\right) »$ при известном напряжении питания статора $\left(u_{s}\right)$, представленная в [18], приведена в (2):

$$
\left\{\begin{array}{l}
\frac{d i_{s A}}{d t}=\frac{1}{x_{s} \sigma}\left(u_{s A}-r_{s} i_{s A}-k_{r} \frac{d \psi_{r A}}{d t}\right) ; \\
\frac{d i_{s B}}{d t}=\frac{1}{x_{s} \sigma}\left(u_{s B}-r_{s} i_{s B}-k_{r} \frac{d \psi_{r B}}{d t}\right) ; \\
\frac{d i_{s C}}{d t}=\frac{1}{x_{s} \sigma}\left(u_{s C}-r_{s} i_{s C}-k_{r} \frac{\left.d \psi_{r C}\right)}{d t}\right) ; \\
\frac{d \psi_{r A}}{d t}=\alpha_{r} i_{s A}-\alpha_{r} \psi_{r A}+\frac{\omega}{\sqrt{3}}\left(\psi_{r C}-\psi_{r B}\right) ; \\
\frac{d \psi_{r C}}{d t}=\alpha_{r}^{\prime} i_{s B}-\alpha_{r} \psi_{r B}+\frac{\omega}{\sqrt{3}}\left(\psi_{r B}-\psi_{r A}\right) ; \\
M=\frac{k_{r}}{\sqrt{3}}\left[\psi_{r A}\left(i_{s B}-i_{s C}\right)-\frac{\omega}{\sqrt{3}}\left(\psi_{r A}-\psi_{r B}\right) ;\right. \\
M-M_{\mathrm{c}}=J_{\Sigma} \frac{d \omega}{d t}+\frac{\omega^{2}}{2} \cdot \frac{d J_{\Sigma}}{d \varphi} ; \frac{d \varphi}{d t}=\omega,
\end{array}\right.
$$

где

$$
\sigma=1-x_{m}^{2} /\left(x_{s} \cdot x_{r}\right), \alpha_{r}=r_{r} / x_{r}, k_{r}=x_{m} / x_{r}, \alpha^{\prime}=\alpha_{r} / \sigma
$$

- электрические параметры двигателя; $\omega, \varphi,-$ угловая скорость и угол поворота вала двигателя; $M$ - момент 
двигателя; $M c, J_{\Sigma}-$ приведённые к валу двигателя момент сопротивления и момент инерции механизма.

Точное кинематическое поведение наземной части привода насоса можно представить с помощью принципиальной схемы, показанной на рис. 3.

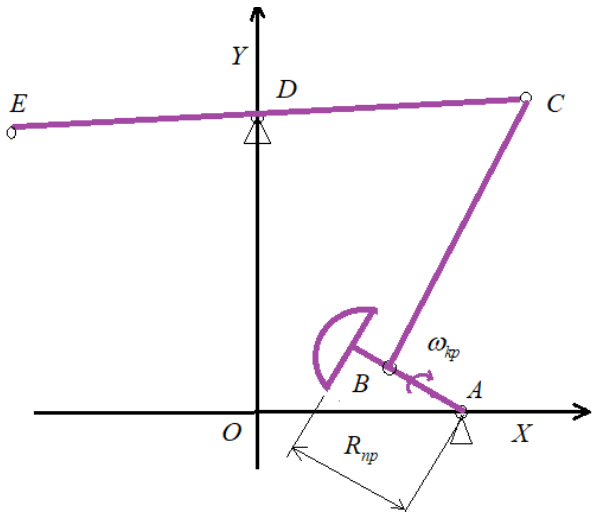

Рис. 3. Кинематическая схема привода штангового насоса

Fig. 3. Kinematic scheme of sucker rod pump

Пусть $A B=r, B C=k, C D=l_{1}, D E=l_{2}, O D=n_{y}$ и $O A=m_{x}$. В любой момент координат точек $A, B, C, D$ и $E$ можно описать формулой (3). Положение и скорость точки подвеса полированного штока определяется через координату $E$.

$$
\left\{A\left(m_{x}, 0\right) ; B\left(x_{B}, y_{B}\right) ; C\left(x_{C}, y_{C}\right) ; D\left(0, n_{y}\right) ; E\left(x_{c}, y_{c}\right)\right\},
$$

где

$$
\begin{gathered}
x_{B}=m_{x}+r \cdot \cos \left(\varphi_{k}\right) ; y_{B}=r \cdot \sin \left(\varphi_{k}\right) ; \\
x_{c}=\sqrt{l_{1}^{2}-\left(y_{c}-n_{y}\right)^{2}} ; y_{c}=\frac{-\beta \pm \sqrt{\beta^{2}-4 \alpha \gamma}}{2 \alpha} ; \\
x_{E}=-x_{C} \frac{l_{2}}{l_{1}} ; y_{E}=n_{y}-\left(y_{C}-n_{y}\right) \frac{l_{2}}{l_{1}} ; \\
\alpha=4\left(\left(n_{y}-y_{B}\right)^{2}+x_{B}^{2}\right) ; \beta=4\left(d\left(n_{y}-y_{B}\right)-2 x_{B}^{2} n_{y}\right) ; \\
\gamma=d^{2}-\left(2 x_{B} l_{1}\right)^{2}+\left(2 x_{B} n_{y}\right)^{2} ; \\
d=x_{B}^{2}-k^{2}+l_{1}^{2}-n_{y}^{2}+y_{B}^{2} ;
\end{gathered}
$$

$\varphi_{k}-$ угловое перемещение кривошипа.

Уравнение движения, описывающее динамику электропривода штангового насоса, нелинейно. Момент сопротивления нагрузки $M_{c}$ и момент инерции механизма $J_{\Sigma}$ являются функциями угла поворота кривошипа. Их описание приведено в (4) и (5) соответственно:

$$
\begin{gathered}
M_{\mathrm{c}}=\sum_{i=1}^{i=q} M_{\mathrm{c} i} u_{i}+\sum_{j=1}^{j=p} F_{\mathrm{c} j} \rho_{j} ; \\
J_{\Sigma}=\sum_{i=1}^{i=n} J_{i} u_{i}^{2}+\sum_{j=1}^{j=k} m_{j} \rho_{j}^{2},
\end{gathered}
$$

где $M_{c i}, F_{c j}$ - момент сопротивления и сила, приложенные к $i, j$-му звену механической части электропривода; $J_{i}, m_{j}$ - момент инерции и масса $i, j$-го звена механической части электропривода; $u_{i}, \rho_{j}$ - передаточная функция и радиус приведения $i, j$-того звена.

\section{Модель насоса}

Плунжер, корпус-цилиндр, всасывающий и нагнетательный клапаны образуют собственно погружной насос. В цикле работы насоса последовательно происходит расширение газа при всасывании жидкости, сжатие газа и нагнетание жидкости. Состояние всасывающего клапана зависит от значения давления на приеме насоса относительно давления нагнетания. Точно так же состояние нагнетательного клапана зависит от значения давления нагнетания насоса относительно давления всасывания. Насос наполняется при открытии всасывающего клапана и опорожняется при открытии напорного клапана.

Во время работы некоторые газы выделяются из пластовой жидкости или растворяются в ней, всасываются или просачиваются в насос вместе с жидкостью. В результате масса и объем газа в насосе постоянно изменяются. Хотя давление насоса трудно измерить, но его значение или скорость изменения можно приблизительно оценить на каждой фазе. Во время фазы заполнения давление насоса принимается равным давлению всасывания, тогда как во время нагнетания жидкости давление насоса равно давлению нагнетания. Однако во время фазы сжатия и расширения изменение давления подчиняется правилам уравнения газового состояния. На основании правила нестационарного изменения давления жидкости в насосе [19] скорость изменения давления жидкости описывается формулой (6), когда плунжер движется вверх. Однако, если плунжер движется вниз, скорость изменения давления жидкости в насосе описывается (7). Нагрузка насоса $\left(F_{p}\right)$ определяется по $(8)$, это весьма важная компонента, поскольку она сильно влияет на величину нагрузки на полированный шток.

$$
\begin{aligned}
& \frac{d p_{H A C}}{d t}= \\
& = \begin{cases}\frac{\left[v_{p l} A_{p l}-\frac{\pi D_{p l} \delta^{3}}{12 \mu L_{p}}\left(P_{d}-p_{H A C}\right)\right] P_{d}}{V_{o g}(-1 / n)\left(p_{H A C} / P_{d}\right)^{-\frac{1}{n}-1}} ; & p_{H A C}>P_{s} ; \\
0 ; & p_{H A C}=P_{s},\end{cases} \\
& \frac{d p_{H A C}}{d t}= \\
& = \begin{cases}\frac{\left[v_{p l} A_{p l}-\frac{\pi D_{p l} \delta^{3}}{12 \mu L_{p}}\left(P_{d}-p_{H A C}\right)\right] P_{s}}{V_{g}(-1 / n)\left(p_{H A C} / P_{s}\right)^{-\frac{1}{n}-1}} ; & p_{H A C}>P_{d} ; \\
0 ; & p_{H A C}=P_{d},\end{cases} \\
& F_{p}(t)=A_{p l}\left(P_{d}-p_{H A C}(t)\right)-A_{r} P_{d},
\end{aligned}
$$

где $v_{p l}$ - скорость плунжера; $V_{o g}$ - объем остаточного газа в цилиндре насоса; $V_{g}$ - объем свободного газа; $\delta$ - зазор между плунжером насоса и цилиндром; $\mu-$ кинематическая вязкость жидкости; $n$ - степенной коэффициент, $A_{p l}$ - площадь поперечного сечения плунжера, $A_{r}$ - площадь поперечного сечения штока, $P_{d}$ - давление нагнетания. 


\section{Модель приточного коллектора}

Приток жидкости в скважину описывается с использованием концепции закона Дарси [3]:

$$
\frac{q}{A}=-\frac{k}{\mu} \frac{d p}{d l},
$$

где $q$ - дебит жидкости; $A$ - площадь поперечного сечения; $\mu$ - вязкость жидкости; $k$ - эффективная проницаемость; $d p / d l-$ градиент давления.

\section{Материальный баланс в скважине}

Состояние приточного коллектора связано с динамикой колонны насосных штанг через динамический уровень жидкости в скважине. Изменение динамического уровня влияет на материальный баланс в скважине. В периоды, когда нагнетательный клапан закрыт, динамический уровень увеличивается на величину, определяемую скоростью притока. С другой стороны, динамический уровень уменьшается на величину, определяемую скоростью притока и оттока. При расчете материального баланса учитываются физические свойства пластовой жидкости, такие как коэффициент объема пласта нефти, соотношение газойля в растворе, коэффициент изотермической сжимаемости, вязкость нефти и т. д., которые описаны в [20].

\section{Имитационные исследования}

Компьютерные методы диагностики требуют большого количества обучающих данных, которые описывают различные рабочие состояния контролируемого оборудования [9]. Однако в течение срока службы скважины возникает лишь несколько возможных рабочих состояний. Более того, образцы от разных скважин не всегда имеют одинаковое распределение, поэтому их прямое использование в качестве обучающих данных вряд ли может дать хорошие результаты. Динамический симулятор штангового насоса позволяет формировать обучающий набор, отражающий различные неисправные рабочие состояния. Чаще всего отказы в работе ШГНУ возникают из-за неисправностей в подземной части. Изменяя параметры в модели коллектора, свойства жидкости или предполагая механические проблемы, можно смоделировать различные возможные сценарии работы ШГНУ. На рис. 4 показаны ваттдиаграммы двигателя, рассчитанные симулятором ШГНУ (рис. 2) для ряда характерных случаев: нормального рабочего состояния; при утечке всасывающего клапана; воздействии газа; недостаточной подачи жидкости; состояниях, когда плунжер ударяет в корпус насоса в верхней (высокая посадка плунжера) или нижней (низкая посадка плунжера) мертвой точке.

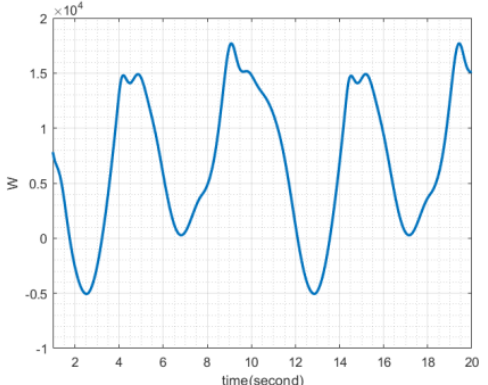

A

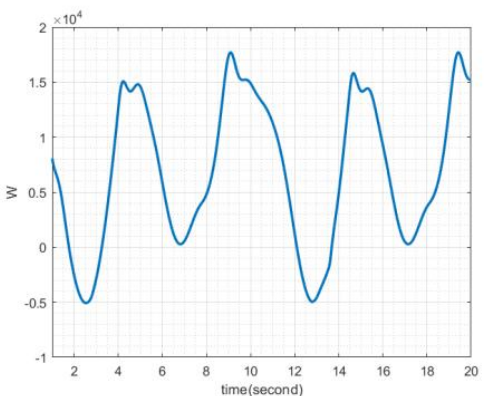

$\mathrm{D}$

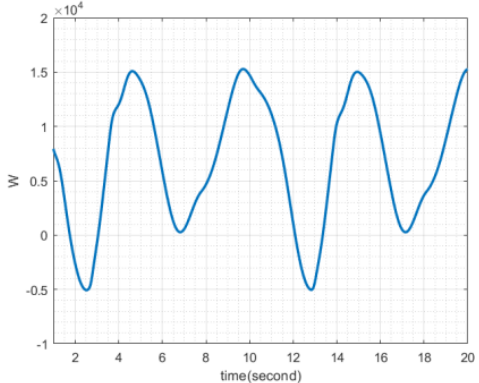

B

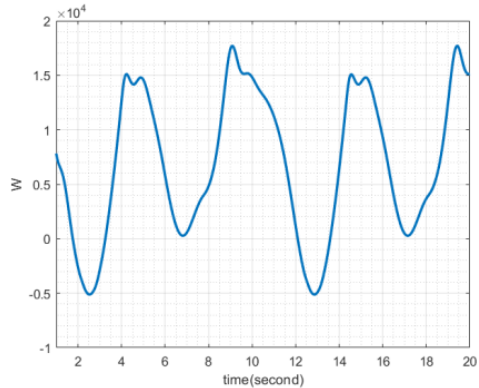

$\mathrm{E}$

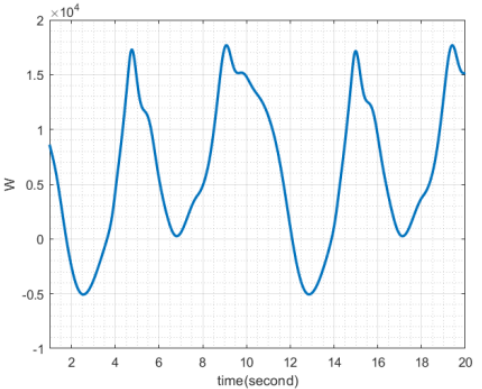

C

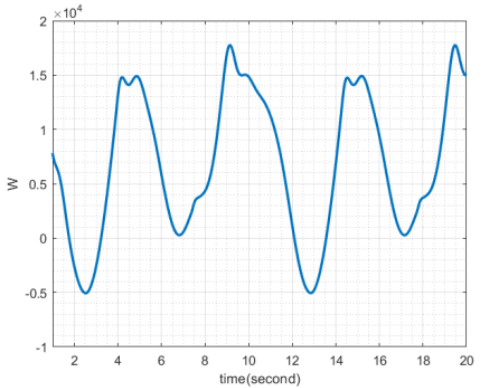

$\mathrm{F}$

Рис. 4. Диаграммы мощности двигателя: А) нормальное рабочее состояние; В) утечка в приемном клапане; C) влияние газа; D) незаполнение насоса; E) высокая посадка плунжера; F) низкая посадка плунжера

Fig. 4. Motor power curves: A) normal working state; B) travelling valve leakage; C) gas effect; D) insufficient liquid supply; E) plunger hit top dead center; F) plunger hit bottom dead center

Очевидно, форма диаграммы мощности двигателя изменяется в ответ на эффект, вызванный соответствующей проблемой. Однако дать оценку текущему рабочему состоянию штангового насоса при визуальном контроле ваттдиаграммы непросто. В таком случае предлагается использовать компьютерные методы распознавания образов. Для компьютерных методов любая выборка должна быть представлена вектором признаков. Вектор признаков должен содержать только полезную информацию и однозначно характеризовать образец. Полезная информация о работе штангового насоса на диаграмме мощности двигателя лучше всего характеризуется значениями мощности в момент переключения клапанов. В данном исследо- 
вании для построения вектора признаков вычисляются следующие значения:

- расстояние между точками переключения клапанов $L_{a}, L_{b}, L_{c}$, как показано на рис. 5 ;

- относительный рабочий коэффициент четырех частей цикла $\left(w_{1}, w_{2}, w_{3}, w_{4}\right)$

$$
\begin{gathered}
w_{1}=\frac{1}{W} \int_{t_{o}}^{t_{1}} P(t) \cdot d t ; \quad w_{2}=\frac{1}{W} \int_{t_{1}}^{t_{2}} P(t) \cdot d t ; \\
w_{3}=\frac{1}{W} \int_{t_{2}}^{t_{3}} P(t) \cdot d t ; w_{4}=\frac{1}{W} \int_{t_{3}}^{t_{4}} P(t) \cdot d t ; \\
W=\int_{t_{0}}^{t_{4}} P(t) \cdot d t,
\end{gathered}
$$

где $t_{0}-t_{1}$ - длительность первой части хода вниз; $t_{1}-t_{2}-$ вторая часть хода вниз; $t_{2}-t_{3}-$ первая часть хода вверх; $t_{3}-t_{4}-$ вторая часть хода вверх; $t_{0}-t_{4}-$ продолжительность цикла.

- относительное значение разницы между двумя пиками $(D)$;

$$
D=\frac{\delta_{1}-\delta_{2}}{\max \left(\delta_{1}, \delta_{2}\right)}
$$

где $\delta_{1}$ - максимальная мощность во время хода вниз, а $\delta_{2}-$ максимальное значение мощности во время хода вверх;

- отношение отрицательной энергии в цикле к положительной $\left(R_{N P}\right)$;

- энергия дисбаланса механизма при движении штока вверх и вниз $\left(w_{u n}\right)$.

$$
w_{u n}=w_{1}+w_{2}-w_{3}-w_{4} .
$$

Средние значения вектора признаков, вычисленные для данных образцов при вариации состояний, показаны в таблице. Оценивая их, можно сказать, что полученные значения однозначно характеризуют каждое рабочее состояние насоса и могут быть использованы для обучения системы диагностики.

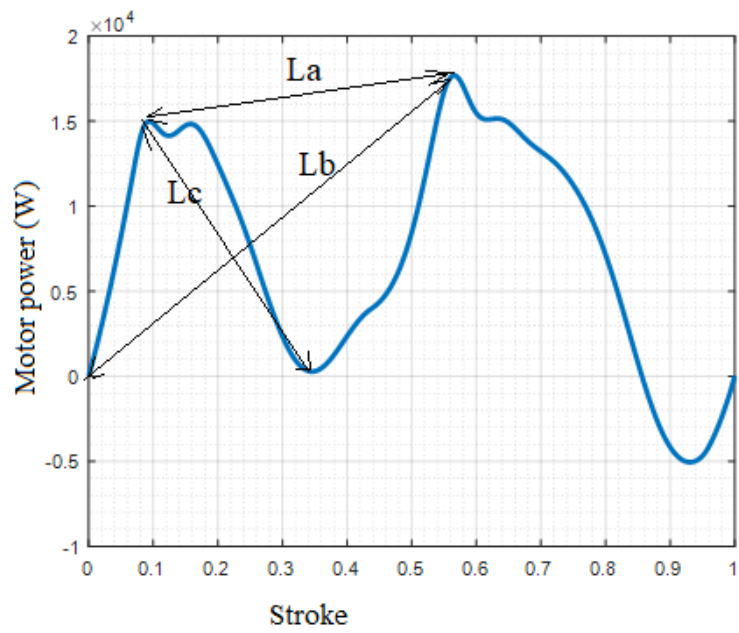

Pис. 5. Образец диаграммы мощности двигателя

Fig. 5. Sample of motor power curve

Таблица. Средние значения вектора признаков для данных образцов

Table. $\quad$ Average value for the given samples

\begin{tabular}{|c|c|c|c|c|c|c|c|c|c|c|}
\hline State & $\mathrm{L}_{\mathrm{a}}$ & $\mathrm{L}_{\mathrm{b}}$ & $\mathrm{L}_{\mathrm{c}}$ & $\mathrm{D}$ & $\mathrm{R}_{\mathrm{NP}}$ & $\mathrm{W}_{1}$ & $\mathrm{~W}_{2}$ & $\mathrm{~W}_{3}$ & $\mathrm{~W}_{4}$ & $\mathrm{~W}_{\mathrm{un}}$ \\
\hline $\mathrm{N}$ & 0,4251 & 0,7967 & 0,4529 & $-0,1417$ & 0,0823 & 0,2018 & 0,1839 & 0,3971 & 0,2172 & 0,2286 \\
\hline TVL & 0,4774 & 0,7297 & 0,4624 & $-0,0443$ & 0,0716 & 0,2409 & 0,1873 & 0,3546 & 0,2172 & 0,1435 \\
\hline GA & 0,4162 & 0,7985 & 0,5027 & $-0,0270$ & 0,1177 & 0,1017 & 0,2036 & 0,4476 & 0,2470 & 0,3893 \\
\hline ILS & 0,3983 & 0,7930 & 0,4502 & $-0,1133$ & 0,1056 & 0,0540 & 0,2027 & 0,4862 & 0,2572 & 0,4867 \\
\hline PHT & 0,4417 & 0,7105 & 0,4374 & $-0,1204$ & 0,1054 & 0,1854 & 0,1835 & 0,4091 & 0,2220 & 0,2622 \\
\hline PHB & 0,4364 & 0,7017 & 0,4340 & $-0,1289$ & 0,0992 & 0,2035 & 0,1867 & 0,3880 & 0,2218 & 0,2195 \\
\hline
\end{tabular}

$N$ - нормальная работа; TVL - утечка приемного клапана; GA - влияние газа; ILS - незаполнение насоса; PHT высокая посадка плунжера; РНВ - низкая посадка плунжера.

$N$ - normal; TVL - travelling valve leakage; GA-gas effect; ILS - insufficient liquid supply; PHT - condition when plunger hits top dead center; $P H B$ - condition when plunger hits bottom dead center.

SVM является машиной принятия решений и не требует апостериорных вероятностей [21]. Поэтому его удобство в использовании данных, сгенерированных моделью [22], очевидно. В предлагаемом исследовании классификатор SVM, созданный обучением на 72 образцах на основе алгоритма SVM, правильно классифицировал по 12 тестовых образцов нормального рабочего состояния ШГНУ, влияние газа и утечку приемного клапана. Однако из 12 тестовых образцов незаполнения насоса 2 образца были классифицированы как нормальная работа, а 3 образца - как влияние газа.

\section{Заключение}

Мониторинг и диагностика штанговых глубинных насосных установок является важной задачей обеспечения их эффективной работы. Информация о рабочем состоянии штангового насоса отражается в динамо- грамме механизма и ваттдиаграмме приводного двигателя. Анализ вида ваттдиаграммы позволяет оценить рабочее состояние штангового насоса, но определить его визуально довольно затруднительно. Кроме того, ручная диагностика неисправностей утомительна и трудоемка. Автоматическая диагностика неисправностей, основанная на методах машинного обучения, представляет особый интерес для разработчиков систем мониторинга. Однако методы машинного обучения требуют большого количества обучающих выборок для обеспечения достаточной точности диагностики. При этом диаграмму мощности двигателя необходимо соотносить с динамограммой, чтобы получить маркированный образец. Таким образом, потребность в большом количестве маркированных образцов для обучения систем диагностики оборудования - это новый вызов для исследователей. Для удовлетворения требований к обучению предлагается динамический 
симулятор электропривода штанговой глубинной насосной установки [23]. Рассмотренный симулятор позволяет генерировать ваттдиаграммы электроприво-

\section{СПИСОК ЛИТЕРАТУРЫ}

1. Tripp H. A review: analyzing beam-pumped wells // Journal of Petroleum Technology. - 1989. - V. 4. - № 5. - P. 457-458.

2. Boyuan Zheng, Xianwen Gao Diagnosis of sucker rod pumping based on dynamometer card decomposition and hidden Markov model // Transactions of the Institute of Measurement and Control. - 2018. - V. 40. - № 16. - P. 4309-4320.

3. Takács Gábor. Sucker rod pumping handbook. - Waltham: Gulf Professional Publishing, 2015. $-585 \mathrm{p}$

4. Ao Zhang, Xianwen Gao. Fault diagnosis of sucker rod pumping systems based on curvelet transform and sparse multi-graph regularized extreme learning machine // International Journal of Computational Intelligence Systems. - 2018. - V. 11. - № 1. P. 428-437.

5. Boyuan Zheng, Xianwen Gao, Xiangyu Li. Diagnosis of sucker rod pump based on generating dynamometer cards // Journal of Process Control - 2019. - V. 77. - P. 76-88.

6. Using the curve moment and the PSO-SVM method to diagnose downhole conditions of a sucker rod pumping unit / Kun Li, Xianwen Gao, Zhongda Tian, Zhixue Qiu // Petroleum Science. 2013. - V. 10. - № 1. - P. 73-80.

7. Multiple fault diagnosis of down-hole conditions of sucker-rod pumping wells based on Freeman chain code and DCA / Kun Li, Xian-wen Gao, Weibing Yang, Yinglong Dai, Zhongda Tian // Petroleum Science. - 2013. - V. 10. - № 3. - P. 347-360.

8. Boyuan Zheng, Xian-wen Gao, Xiangyu Li. Fault detection for sucker rod pump based on motor power // Control Engineering Practice. - 2019. - V. 86. - P. 37-47.

9. Zheng B., Gao X., Pan R. Sucker rod pump working state diagnosis using motor data and hidden conditional random fields // IEEE Transactions on Industrial Electronics. - 2020. - V. 67. № 9. - P. 7919-7928.

10. Зюзев А.М., Бубнов М.В. Диагностика уравновешенности штанговой глубинной насосной установки по ваттметрограмме // Известия Томского политехнического университета. Инжиниринг георесурсов. - 2019. - Т. 330. - № 4. - С. 178-187.

11. Weicheng Li, Shimin Dong, Xiurong Sun. An improved sucker rod pumping system model and swabbing parameters optimized design // Mathematical Problems in Engineering. - 2018. V. 2018. - P. 1-15. да для различных типов неисправностей насоса, которые могут быть использованы для обучения систем автоматической диагностики.

12. Зюзев А.М. Математические модели механической части электроприводов. - Екатеринбург: УрФУ, 2010. - 154 с.

13. Takács G. Sucker-rod pumping manual. - Tulsa, Oklahoma: PennWell Books, 2003. - 359 p.

14. Выбор критериев уравновешивания штанговых скважинных насосных установок / Г.И. Бикбулатова, Г.И. Болтнева, А.С. Галеев, Р.Н. Сулейманов, С.Л. Сабанов // Газовая промышленность. - 2019. - № 11 (792). - С. 124-128.

15. Identification of working condition from sucker-rod pumping wells based on multi-view co-training and Hessian regularization of SVM / B. Zhou, Y. Wang, W. Liu, B. Liu // 2018 14th IEEE International Conference on Signal Processing (ICSP). - Beijing, China, 2018. - P. 969-973.

16. Everitt T., Jennings J.W. An improved finite-difference calculation of downhole dynamometer cards for sucker-rod pumps // SPE Production Engineering. - 1992. - V. 7. - P. 121-127.

17. Lv W.J., Xing M. Characteristic analysis of longitudinal vibration sucker rod string with variable equivalent stiffness // MATEC Web Conf. - Kuala Lumpur, Malaysia, 2018. - V. 153. - P. 1-5.

18. Фираго Б.И., Павлячик Л.Б. Регулируемые электроприводы переменного тока. - Мн.: Техноперспектива, 2006. 363 с.

19. Xing M. Response analysis of longitudinal vibration of sucker rod string considering rod buckling // Advances in Engineering Software. - 2016. - V. 99. - P. 49-58

20. McCain Jr. W.D. The properties of petroleum fluids. - Tusla, Oklahoma: PennWell Publishing Company, 1990. - 548 p.

21. Bishop Ch.M. Pattern recognition and machine learning. - New York: Springer, 2006. $-738 \mathrm{p}$.

22. Semenov A.V., Tecle S.I., Ziuzev A. Modeling induction motor driven sucker rod pump in MATLAB simscape // 2020 Russian Workshop on Power Engineering and Automation of Metallurgy Industry: Research and Practice (PEAMI). - Magnitogorsk, Russia, 2020. - P. 67-71.

23. Семенов А.В., Зюзев А.М., Текле С.И. Симулятор штанговой глубинно насосной установки: свидетельство о государственной регистрации программы для ЭВМ № 2020666580, 11.12.2020, заявка № 2020665912, 4 дек 2020. Федеральный институт промышленной собственности.

Поступила 13.01.2022 г.

\section{Информация об авторах}

Зюзев A.M., доктор технических наук, профессор кафедры электропривода и автоматизации промышленных установок Уральского федерального университета.

Самуэль Исаак Текле, аспирант кафедры электропривода и автоматизации промышленных установок Уральского федерального университета. 
UDC 622.276 .53

\title{
SUCKER ROD PUMPING SYSTEM: CHALLENGES TO DEVELOP DIAGNOSTIC SYSTEM AND ROLE OF DYNAMIC SIMULATOR
}

\author{
Anatolii M. Ziuzev'1, \\ a.m.zyuzev@urfu.ru \\ Samuel Isaac Tecle ${ }^{1}$, \\ samuel47tecle@gmail.com \\ 1 Ural Federal University, \\ 19, Mira street, Yekaterinburg, 620002, Russia.
}

Relevance. Production benefits and efficiency of sucker rod pump installations is highly dependent on the accuracy of monitoring and fault diagnosis system used. When sucker rod pump is operated at faulty working states, the rate of equipment failure increases and the production efficiency decreases. Moreover, since the sucker rod pump operates deep in the underground, maintenance cost is more, and production is interrupted for longer time. Hence, improving monitoring and diagnostic system for sucker rod pump operation has become very important. The information about sucker rod pump working state is embodied in the dynamometer card and motor power curve. Monitoring sucker rod pump using motor power curve is more advantageous than dynamometer card. It can be used to monitor both surface and subsurface equipment. Moreover, motor power curve is obtained using more reliable current and voltage measurements. Therefore, the motor power curve provides a better alternative evidence for development of monitoring and diagnostic systems for sucker rod pumps.

The main aim of the research is to ease the challenges those impede the promotion of diagnostic models using motor power curve.

Objects: electrical drive, sucker rod pumping unit, oil producing well.

Methods: sucker rod pump simulation model; feature extraction method that produces a feature vector to uniquely represent each working state; diagnostic method based on support vector machine.

Results. 72 labeled motor power curves representing six working states namely: normal working state, travelling valve leakage, gas affected, insufficient liquid supply, condition when the plunger hits top dead center and bottom dead center, are generated. It was seen that the feature vector constructed based on the valve working points and energy consumption represent uniquely each working state. It was also seen that the support vector machine classifier correctly classifies the samples for normal, travelling valve leakage, gas affected working states. However, some samples of insufficient liquid supply were misclassified as gas affected and normal.

\section{Key word:}

Sucker rod pump, feature extraction, dynamometer card, fault diagnosis.

\section{REFERENCE}

1. Tripp H. A review: analyzing beam-pumped wells. Journal of Petroleum Technology, 1989, vol. 41, no. 5, pp. 457-458

2. Boyuan Zheng, Xianwen Gao. Diagnosis of sucker rod pumping based on dynamometer card decomposition and hidden Markov model. Transactions of the Institute of Measurement and Control, 2018, vol. 40, no. 16, pp. 4309-4320.

3. Takács Gábor. Sucker rod pumping handbook. Waltham, Gulf Professional Publishing, 2015. 585 p.

4. Ao Zhang, Xianwen Gao. Fault diagnosis of sucker rod pumping systems based on curvelet transform and sparse multi-graph regularized extreme learning machine. International Journal of Computational Intelligence Systems, 2018, vol. 11, no. 1, pp. 428-437.

5. Boyuan Zheng, Xianwen Gao, Xiangyu Li. Diagnosis of sucker rod pump based on generating dynamometer cards. Journal of Process Control, 2019, vol. 77, pp. 76-88.

6. Kun Li, Xianwen Gao, Zhongda Tian, Zhixue Qiu. Using the curve moment and the PSO-SVM method to diagnose downhole conditions of a sucker rod pumping unit. Petroleum Science, 2013, vol. 10 , no. 1 , pp. $73-80$.

7. Kun Li, Xianwen Gao, Weibing Yang, Yinglong Dai, Zhongda Tian. Multiple fault diagnosis of down-hole conditions of suckerrod pumping wells based on Freeman chain code and DCA. Petroleum Science, 2013, vol. 10, no. 3, pp. 347-360.

8. Boyuan Zheng, Xianwen Gao, Xiangyu Li. Fault detection for sucker rod pump based on motor power. Control Engineering Practice, 2019, vol. 86, pp. 37-47.

9. Zheng B., Gao X., Pan R. Sucker rod pump working state diagnosis using motor data and hidden conditional random fields. IEEE Transactions on Industrial Electronics, 2020, vol. 67, no. 9, pp. 7919-7928.

10. Zyuzev A.M., Bubnov M.V. Diagnostics of the balance of the rod deep pumping unit according to the wattmetrogram. Bulletin of the
Tomsk Polytechnic University. Geo Assets Engineering, 2019, vol. 330, no. 4, pp. 178-187. In Rus.

11. Weicheng Li, Shimin Dong, Xiurong Sun. An improved sucker rod pumping system model and swabbing parameters optimized design. Mathematical Problems in Engineering, 2018, vol. 2018, pp. $1-15$.

12. Ziuzev A.M. Matematicheskie modeli mekhanicheskoy chasti elektroprivodov [Mathematical models of the mechanical part of electric drives]. Ekaterinburg, URFU Publ., 2010. 154 p.

13. Takács G. Sucker-rod pumping manual. Tulsa, Oklahoma, PennWell Books, 2003. $359 \mathrm{p}$.

14. Bikbulatova G.I., Boltneva G.I., Galeev A.S., Suleymanov R.N., Sabanov S.L. Selection of the balance criterion for rod borehole pumping units. Gas industry, 2019, no. 11 (792), pp. 124-128.

15. Zhou B., Wang Y., Liu W., Liu B. Identification of working condition from sucker-rod pumping wells based on multi-view cotraining and Hessian regularization of SVM. 2018 14 $4^{\text {th }}$ IEEE International Conference on Signal Processing (ICSP). Beijing, China, 2018. pp. 969-973.

16. Everitt T., Jennings J.W. An improved finite-difference calculation of downhole dynamometer cards for sucker-rod pumps. SPE Production Engineering, 1992, vol. 7, pp. 121-127.

17. Lv W.J., Xing M. Characteristic analysis of longitudinal vibration sucker rod string with variable equivalent stiffness. MATEC Web Conf. - Kuala Lumpur, 2018, vol. 153, pp. 1-5.

18. Firago B.I., Pavlyachik L.B. Reguliruemye elektroprivody peremennogo toka [Adjustable AC electric drives]. Minsk, Tekhnoperspektiva Publ., 2006. 363 p.

19. Xing M. Response analysis of longitudinal vibration of sucker rod string considering rod buckling. Advances in Engineering Software, 2016, vol. 99, pp. 49-58.

20. McCain Jr. W.D. The properties of petroleum fluids. Tusla, Oklahoma, PennWell Publishing Company, 1990. 548 p. 
21. Bishop Ch.M. Pattern recognition and machine learning. New York, Springer, 2006. $738 \mathrm{p}$.

22. Semenov A.V., Tecle S.I., Ziuzev A. Modeling induction motor driven sucker rod pump in MATLAB simscape. 2020 Russian Workshop on Power Engineering and Automation of Metallurgy Industry: Research and Practice (PEAMI). Magnitogorsk, Russia, 2020. pp. 67-71.

\section{Information about the authors}

Anatolii M. Ziuzev, Dr. Sc., professor of Ural Federal University.

Samuel Isaac Tecle, post graduate student, Ural Federal University.
23. Semenov A.V., Ziuzev A., Tecle S.I. Simulyator shtangovoy glubinno nasosnoy ustanovki: svidetelstvo o gosudarstvennoy registratszii programmy dlya EVM [Sucker rod pumping unit simulator: certificate of state registration of a computer program]. RF, no. 2020666580, 2020.

Received: 13 January 2022. 